\title{
Prevalência de sintomas de distúrbios osteomusculares relacionados ao trabalho autorreferido em estudantes e professores de Odontologia
}

\author{
Prevalence of symptoms of musculoskeletal disorders related to self-reported work Dentistry \\ student and professors \\ Prevalencia de sintomas de transtornos musculoesqueléticos relacionados com el trabajo \\ autoinformado en estudiantes y professores de Odontología
}

\section{Resumo}

Os distúrbios osteomusculares relacionados ao trabalho (DORT) é o nome genérico dado a um conjunto de afecções heterogêneas que acometem músculos, articulações e nervos, que pode aparecer em indivíduos submetidos a certas condições de trabalho. Portanto, o presente estudo teve como objetivo verificar a prevalência dos sintomas de DORT em estudantes e professores do curso de odontologia. Para avaliação dos sintomas de DORT foi utilizado a versão brasileira do Questionário Nórdico de Sintomas Osteomusculares (QNSO), aplicado a uma amostra de indivíduos estudantes e professores do curso de odontologia. Para compreensão dos dados, foi realizado análises descritivas dos dados. Quinhentos e cinco $(n=505)$ indivíduos participaram do estudo, sendo $75 \%$ do sexo feminino e $25 \%$ do sexo masculino. Do total de entrevistados 30\% relataram uma jornada de trabalho de seis horas, $51 \%$ uma jornada de até oito horas e 19\% uma jornada de trabalho superior a oito horas. Em relação à dor musculoesquelética, $76 \%$ da amostra relatou dor na região cervical e lombar com certa frequência. Os resultados preliminares do presente estudo sustentam a hipótese de que os sintomas de DORT podem se iniciar logo na graduação de estudantes de odontologia, com frequência nas regiões cervical e lombar.

Palavras-chave: DORT; Ergonomia; Postura; Estudante de odontologia.

\begin{abstract}
Work-related musculoskeletal disorders (WMSD) is the generic name given to a set of heterogeneous affections that affect muscles, joints, and nerves, which can appear in individuals submitted to certain working conditions. Therefore, the present study aimed to verify the prevalence of WMSD symptoms in dentistry students and professors. To assess WMSD symptoms, the Brazilian version of the Nordic Musculoskeletal Symptoms Questionnaire (QNSO) was used, applied to a sample of students and professors from the dentistry course. To understand the data, a descriptive analysis of the data was performed. Five hundred five $(n=505)$ individuals participated in the study, $75 \%$ female and $25 \%$ male. Of the total respondents, 30\% reported a workday of six hours, $51 \%$ a workday of up to eight hours, and $19 \%$ a workday of more than eight hours. Regarding musculoskeletal pain, $76 \%$ of the sample reported pain in the cervical and lumbar region with some frequency. The preliminary results of the present study support the hypothesis that WMSD symptoms may begin as early as the graduation of dentistry students, often in the cervical and lumbar regions. Keywords: WMSD; Ergonomics; Posture; Dentistry student.
\end{abstract}




\begin{abstract}
Resumen
Trastornos musculoesqueléticos relacionados con el trabajo (TMERT) es el nombre genérico que se le da a un conjunto de afecciones heterogéneas que afectan a músculos, articulaciones y nervios, que pueden aparecer en individuos sometidos a determinadas condiciones laborales. Por lo tanto, el presente estudio tuvo como objetivo verificar la prevalencia de síntomas de TMERT en estudiantes y profesores de odontología. Para evaluar los síntomas de TMERT se utilizó la versión brasileña del Cuestionario Nórdico de Síntomas Musculoesqueléticos (QNSO), aplicado a una muestra de estudiantes y profesores del curso de Odontología. Para comprender los datos, se realizó un análisis descriptivo de los datos. Quinientos cinco $(\mathrm{n}=505)$ individuos participaron en el estudio, $75 \%$ mujeres y $25 \%$ hombres. Del total de encuestados, el 30\% informó una jornada laboral de seis horas, el 51\% una jornada laboral de hasta ocho horas y el 19\% una jornada laboral de más de ocho horas. En cuanto al dolor musculoesquelético, el 76\% de la muestra refirió dolor en la región cervical y lumbar con cierta frecuencia. Los resultados preliminares del presente estudio apoyan la hipótesis de que los síntomas de TMERT pueden comenzar desde la graduación de los estudiantes de odontología, a menudo en las regiones cervical y lumbar.
\end{abstract}

Palabras clave: TMERT; Ergonomía; Postura; Estudiante de Odontología.

\title{
1. Introdução
}

O distúrbio osteomuscular relacionado ao trabalho (DORT) é conhecido por apresentar diferentes sintomas em sincronia como, dor localizada, irradiada ou generalizada, desconforto, fadiga, sensação de peso, formigamento, dormência, sensação de diminuição de força, inchaço, enrijecimento muscular entre outros (MS, 2012; Silva et al. 2020). A combinação de sobrecarga estática ou dinâmica das estruturas anatômicas do sistema neuromuscular somadas a movimentos repetitivos são determinantes para o desenvolvimento das doenças musculoesqueléticas (MS, 2012; Hammig \& Bauer, 2014; Sousa et al. 2020).

Na década de 80 foram relatados os primeiros casos de DORT no Brasil, esses estavam relacionados à profissão dos digitadores (Medeiros \& Segatto, 2012). Atualmente, o DORT é a segunda causa de afastamento por doença ocupacional, principalmente entre trabalhadores da indústria (Casarin \& Caria, 2008; Carmo et al. 2011; Medeiros \& Segatto, 2012; Hammig \& Bauer, 2014). Sua etiologia é multifatorial e o diagnóstico se faz através de exame clínico, conhecimento da vida profissional, história da doença atual e exame físico detalhado. Entre os Cirurgiões Dentistas (CD) as principais patologias são as tendinites, tenossinovites, síndrome do túnel do carpo, miosites e bursites (Medeiros \& Segatto, 2012).

Segundo estudo recente, acredita-se que o processo se iniciasse por volta da terceira e quarta década de vida, acelerando na próxima década, no entanto, o desenvolvimento do DORT pode ser potencializado em idades precoces, quando fatores que predispõe estejam presentes (Carvalho, Brasileiro \& Lima, 2019). Um estudo recente verificou maior acometimento das patologias no sexo masculino, principalmente entre os profissionais da indústria devido ao maior número de operadores de máquinas e ajudantes de produção (Santos et al. 2020).

A evolução da doença ocorre em quatro estágios. No primeiro, a dor aparece durante os movimentos e é difusa. No segundo, a dor é mais persistente e localizada e os sintomas melhoram com repouso prolongado. No terceiro, a doença já é crônica, a dor não cede com repouso e é sentida em pontos definidos. No último estágio, os processos inflamatórios podem causar deformidades, cistos, edemas e perda da potência muscular. A dor torna-se insuportável podendo dificultar a prática de atividades triviais do dia a dia (Rosemberg, 2005).

Os fatores desencadeantes gerais têm relação com os movimentos de preensão dos instrumentos, movimentos repetitivos nos procedimentos e com a manutenção da postura estática durante a jornada diária (Sousa et al. 2020). As regiões acometidas região lombar, pescoço, punho/mão/dedos e ombros, e segundo a literatura, entre os estudantes de odontologia a região do pescoço é a mais acometida, seguida das regiões lombar e ombro (Mascarenhas \& Novaes, 2015).

$\mathrm{Na}$ odontologia, o profissional está exposto ao risco em consequência da postura inadequada permanente e mão estável. O fato de sustentar essa postura sem ergonomia, mesmo com equipamentos mais adequados, gera sobrecarga no pescoço, ombro e costas do $\mathrm{CD}$ um desconforto ao longo do trabalho, a execução de movimentos repetitivos, estresse e 
cansaço durante uma extensa jornada (Carmo et al. 2011). A Odontologia é considerada uma profissão "estressante", frequentemente associada aos agravos à saúde, que apresenta importantes fatores de risco para o desenvolvimento de doenças ocupacionais de origem física, química, biológica, ergonômica, mecânica e psíquica (Silva \& Jesus, 2013).

Além disso, é importante ressaltar que embora o CD permaneça na posição sentada, isso não é suficiente para diminuir o desgaste físico da profissão, pois, muitas vezes, os esforços em postura sentada são maiores e seus movimentos tornam-se mais dispendiosos. Sentar durante longos períodos é cansativo para o corpo porque, embora possa tirar o peso dos pés, aumenta a tensão nas costas em até $50 \%$ em relação a permanecer em pé (Silva \& Jesus, 2013). Além disso, o profissional permanece por muito tempo com os membros superiores suspensos, assim como o tronco inclinado para frente, sentado e com a coluna lombar em contratura constante executando rotações do tronco e flexões da cabeça (Borges, Pucci \& Torres, 2009).

Estudos vêm demonstrando que os sintomas relacionados ao desenvolvimento de DORT se iniciam no início da carreira do profissional $\mathrm{CD}$, podendo se desenvolver precocemente durante o período da graduação (Diaz-Caballero et al. 2010; Khan \& Chew, 2013). Portanto, o objetivo do presente estudo foi verificar a prevalência dos sintomas de DORT em estudantes e professores do curso de odontologia.

\section{Métodos}

Trata-se de um estudo descritivo, exploratório, de corte transversal, pois descreve um fenômeno delimitando-se o momento no qual os indivíduos foram avaliados. Este estudo foi previamente aprovado pelo Comitê de Ética em Pesquisa com Seres Humanos (CAAE \#40993514.8.0000.5385). Todos os participantes foram informados sobre os procedimentos do exame e garantidos acerca do sigilo das informações coletadas.

Os seguintes critérios de elegibilidade foram aplicados: (1) estudantes matriculados no curso de odontologia dos períodos integral e noturno; (2) professores do curso de odontologia de ambos os períodos; e (3) consentiram em participar do estudo com o preenchimento do Termo de Consentimento Livre e Esclarecido (TCLE). Foram excluídos aqueles que apresentassem história de doenças articulares, musculares, vasculares ou reumáticas; com má formação congênita ou com sintomas relacionados a outras doenças que não sejam os DORT.

Os dados do presente estudo foram coletados no período entre janeiro e maio de 2015 e contou com a participação de quinhentos e cinco $(n=505)$ indivíduos. Desses, quatrocentos e cinquenta e sete $(n=457)$ eram estudantes de odontologia e cinquenta e oito $(\mathrm{n}=58)$ professores $\mathrm{CD}$ do curso dessa mesma instituição.

Para coleta dos dados referentes aos sintomas de DORT, foi utilizado o Questionário Nórdico de Sintomas Osteomusculares (QNSO), traduzido e validado para população brasileira (Pinheiro, Tróccoli \& Carvalho, 2002). O QNSO é composto por questões referentes aos dados sociodemográficos (sexo, idade, peso, altura, estado civil, preferência manual) e dados ocupacionais (função, tempo de exercício da atividade, jornada de trabalho e hábitos e estilo de vida (exercícios físicos e outras atividades) (Pinheiro, Tróccoli \& Carvalho, 2002). Com base em uma figura do corpo humano com as regiões apontadas, os participantes foram convidados a indicar a frequência em que sentiam dor, dormência, formigamento ou desconforto nas regiões do corpo, nos últimos 12 meses, numa escala de: (0) não; (1) raramente; (2) com frequência e (3) sempre (Pinheiro, Tróccoli \& Carvalho, 2002). Além disso, os participantes foram questionados quais dos sintomas relatados anteriormente estavam relacionados à prática da odontologia (Pinheiro, Tróccoli \& Carvalho, 2002). Os dados foram analisados de forma descritiva, por meio de gráficos de frequência. 


\section{Resultados}

Participaram do estudo quinhentos e cinco ( $\mathrm{n}=505)$ indivíduos, sendo $75 \%$ do sexo feminino $(\mathrm{n}=379)$ e $25 \%$ do sexo masculino (n=126), com idade média de 23.5 anos. Dos participantes do estudo, 9.8\% eram professores do curso de odontologia ( $\mathrm{n}=48)$, enquanto a maioria da população estudada eram estudantes desse mesmo curso $91.2 \%(\mathrm{n}=357)$.

Em relação à jornada de trabalho, 30\% dos participantes relataram uma jornada de trabalho de até 6 horas diárias, enquanto $51 \%$, até oito horas e $19 \%$, mais de oito horas. $77 \%$ dos participantes relataram não realizar atividade profissional extra. Em relação às atividades diárias, $66 \%$ dos participantes relataram realizar serviços domésticos, $76 \%$ utilizavam computadores na sua rotina diária, $38 \%$ relataram realizar atividade física e $20 \%$ realizavam atividades com alta atividade dos membros superiores. Com relação ao lado dominante, $92 \%$ eram destros, $7 \%$ canhotos e $1 \%$ ambidestros.

Entre os professores do curso de odontologia, $54 \%$ dos profissionais relataram ter realizado o planejamento do consultório no que diz respeito a ergonomia. $80 \%$ dos profissionais relataram trabalhar a quatro mãos e somente $15 \%$ dos professores relataram realizar qualquer tipo de alongamento/exercícios durante a jornada de trabalho. De forma subjetiva, $73 \%$ dos profissionais relataram uma qualidade de vida e satisfação com a profissão.

A Figura 1 apresenta os dados em frequência (\%) das dores, divididas por regiões relatadas pelos participantes. Onde foi possível observar que as regiões com maiores taxas de prevalência de sintomas foram a região cervical (75\%) e região lombar (76\%), enquanto as menores taxas foram observadas nas regiões de cotovelos (18\%), antebraços (26\%) e braços (46\%).

Figura 1. Frequência (\%) de dores, divididas por regiões, relatadas pelo total de participantes ( $\mathrm{n}=505)$.

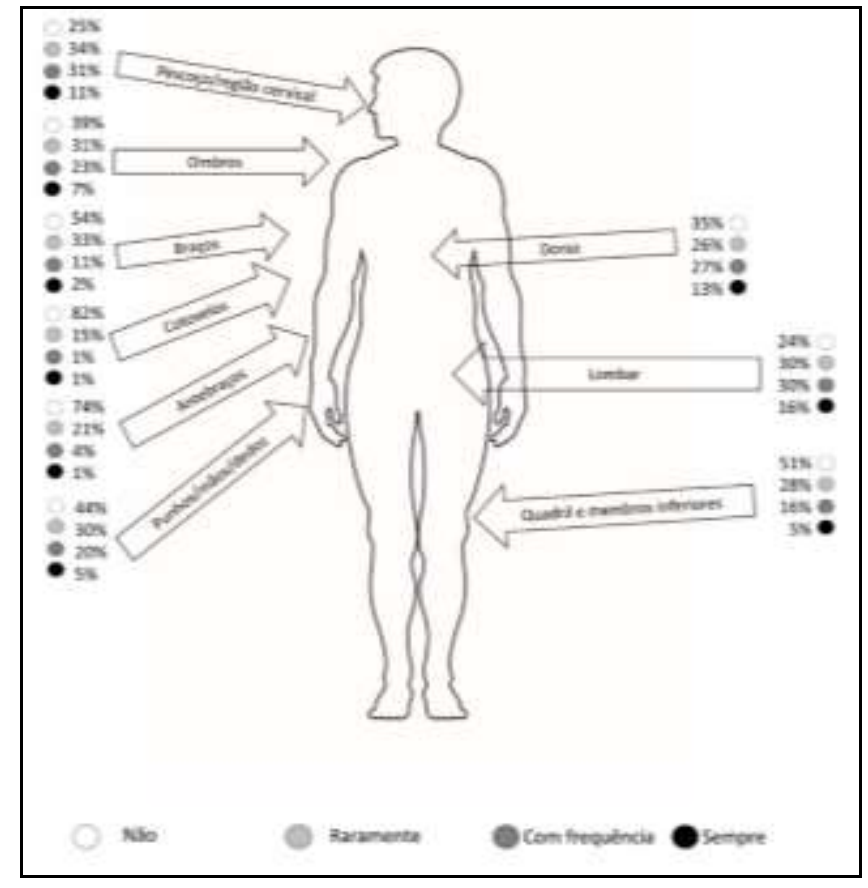

Fonte: Autores.

A Figura 2 apresenta os dados referentes à frequência dos sintomas de DORT de acordo com o sexo. Dos participantes do sexo feminino, 52\% relataram dores na região de pescoço e região cervical, $44 \%$ na região lombar e somente $2 \%$ na região dos cotovelos. Enquanto para os participantes do sexo masculino, $35 \%$ relatam problemas na região de pescoço e região cervical, $34 \%$ na região lombar e também somente $2 \%$ relataram problemas na região dos cotovelos. Entre os sexos feminino e masculino, $16 \%$ e $29 \%$, respectivamente, não relataram nenhum dos sintomas. 
Figura 2. Frequência (\%) dos sintomas de DORT por sexo.

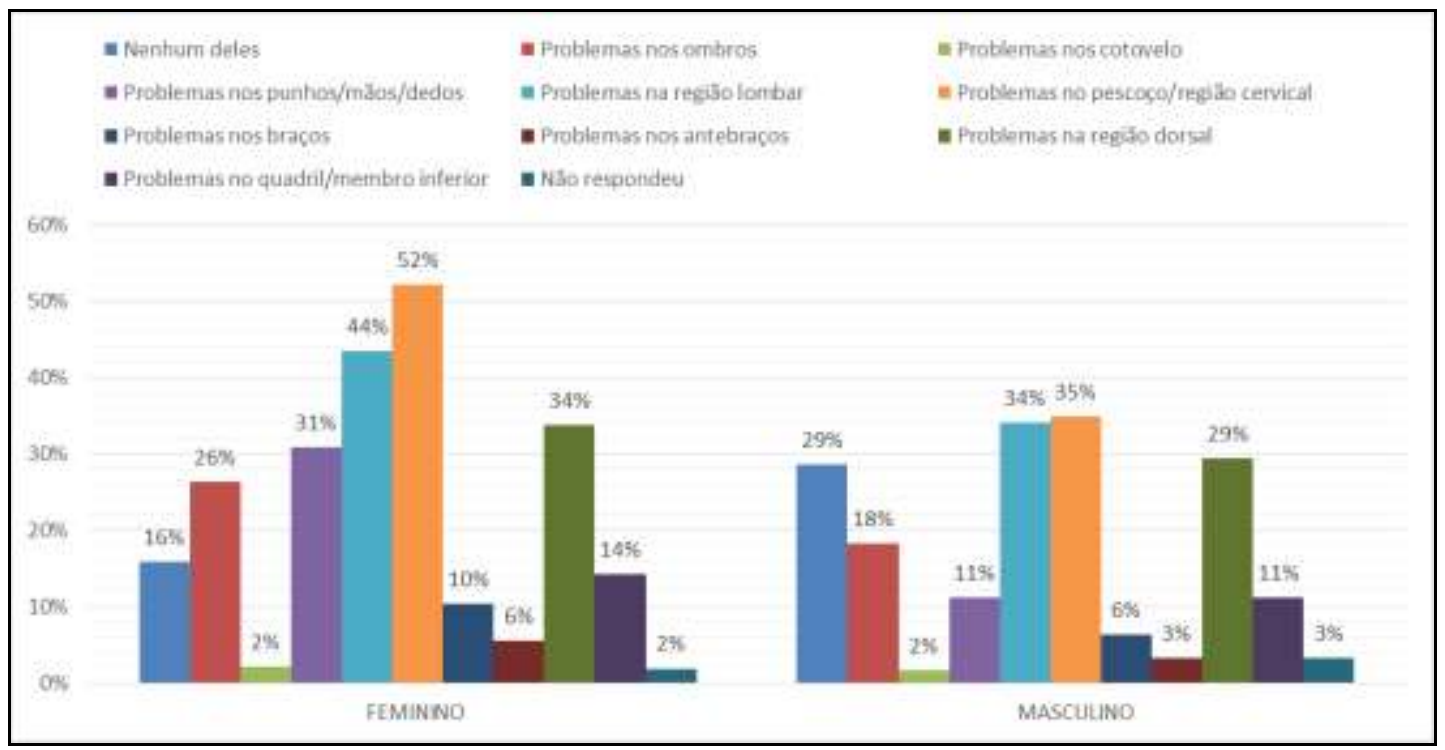

Fonte: Autores.

A Figura 3 apresenta os dados referentes a frequência dos sintomas de DORT de acordo com a faixa etária dos participantes. Para todas as faixas etárias as regiões mais prevalentes foram: região do pescoço e/ou região cervical e região lombar. Enquanto problemas nas regiões de mãos, punhos ou dedos foi mais frequente nos mais jovens. Os sintomas na região dorsal demonstrou menor prevalência nos mais velhos.

Figura 3. Distribuição dos resultados de acordo com a faixa etária.

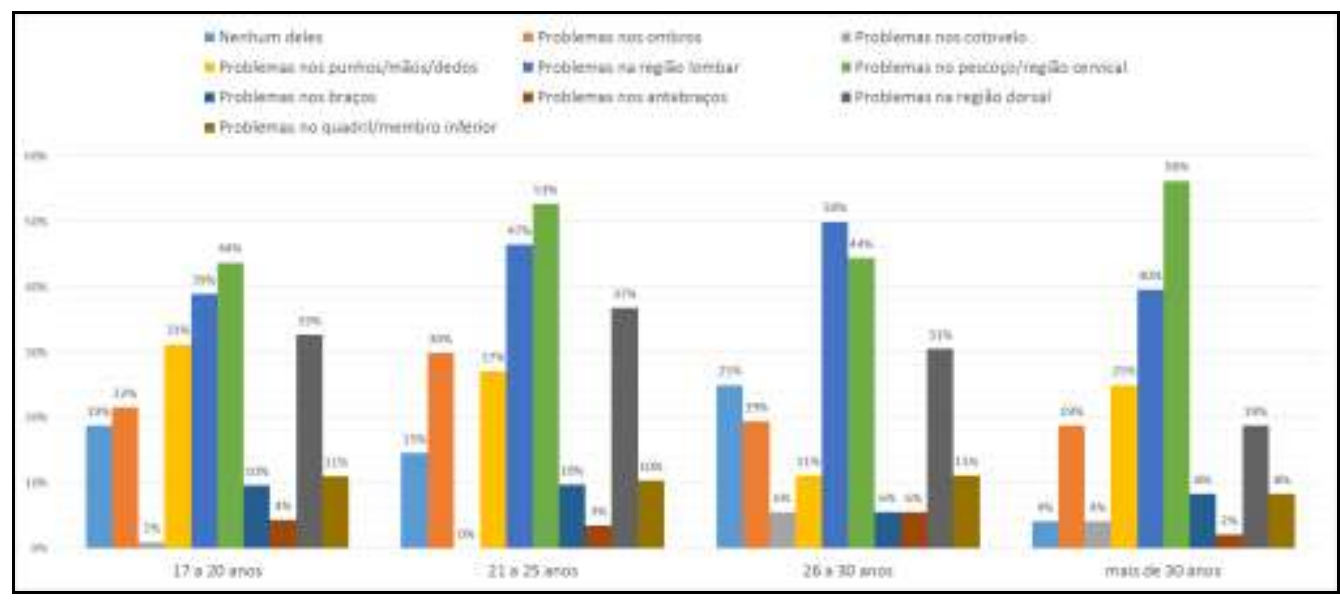

Fonte: Autores.

\section{Discussão}

O objetivo do presente estudo foi verificar a prevalência dos principais sintomas de DORT em indivíduos estudantes e professores de odontologia. Os resultados descritivos e preliminares do estudo verificaram que os sintomas de DORT podem ser relatados desde a graduação de odontologia, sendo a região de pescoço e/ou cervical e a região lombar as mais acometidas. Cerca de $76 \%$ da população em estudo relatou sintomas nessas regiões, e o nosso resultado vem corroborar com estudos prévios da literatura (Carmo et al. 2011; Silva et al. 2013; Silva e Jesus 2013; Mascarenhas e Novaes 2015). 
Os indivíduos do sexo feminino participantes do presente estudo apresentaram maior prevalência de sintomas de DORT quando comparados com indivíduos do sexo masculino, o que foi confirmado em outros estudos (Carmo et al. 2011; Barbosa, Assunção \& Araujo, 2012). Indivíduos do sexo feminino estão mais propensos a desenvolver tais distúrbios, o que está de acordo com a literatura, este fato pode ser justificado pelo predomínio das mulheres na odontologia, além das responsabilidades e atividades repetitivas no exercício da profissão, são responsáveis por outras atividades no dia a dia.

Segundos os resultados, a prevalência de sintomas de DORT quando verificada nos diferentes sexos, foi possível observar que a região cervical, lombar e punhos foram as mais afetadas, enquanto a região de cotovelos a região menos afetada. Na literatura, são relatados resultados semelhantes para estudantes de odontologia, cujas áreas mais afetadas foram coluna cervical, lombar, punho, região torácica, ombro, braços, dedos, antebraço e cotovelo (Siqueira et al. 2010). O fato pode estar relacionado com os movimentos repetitivos durante os procedimentos clínicos e a manutenção da postura estática na jornada de trabalho (Aguiar, Neves \& Araújo, 2014).

Pois, além disso, a posição sentada sem apoio para a coluna lombar, aliada à excessiva flexão de tronco e pescoço com a manutenção dos braços abduzidos e sem apoio está diretamente relacionada com os sintomas de desconforto e/ou dor na região lombar. Segundo estudo recente, as dores lombares estão relacionadas a uma postura incorreta e não confortável, ainda que sua resolução seja voluntária, a exposição a essa postura por um período de tempo, irá ocasionar uma lombalgia crônica diante da degeneração dos discos entre as vértebras (Mattos et al. 2021). Por isso, vale ressaltar a importância da realização de exercícios de alongamento antes, durante e após a jornada de trabalho (Marques et al. 2007).

Com relação à faixa etária, os resultados demonstraram que as áreas mais afetadas foram a região de pescoço e/ou região cervical e região lombar para todas as idades. Os sintomas de DORT nas regiões de mãos, punhos ou dedos foram mais frequentemente relatados por indivíduos mais jovens, o que pode ser explicado pela pouca experiência dos estudantes de odontologia. A maior faixa etária (com mais de 30 anos) foi a que mais relatou a presença de sintomas de DORT, o que vai de encontro com a literatura (Carvalho, Fernandes \& Lima, 2019), além disso, essa faixa etária era composta na sua grande maioria por profissionais CD. Estudos semelhantes apontam que os sintomas de dor aumentam com a idade, principalmente nos profissionais entre a quarta e quinta década de vida. Com relação ao tempo de trabalho do $\mathrm{CD}$, foi observado que quando maior o tempo de profissão, maior a incidência de dor (Regis Filho, Michels \& Sell, 2006).

No entanto, a maioria dos participantes do estudo (85\%) não realizavam algum tipo de exercício ou alongamento antes ou durante a jornada de trabalho, o que pode justificar a prevalência dos sintomas de DORT. Os exercícios ou alongamento podem ajudar a diminuir a dor devido à má postura durante os atendimentos e um estilo de vida saudável, incluindo outras práticas de atividade física, além de melhor organizar a ergonomia, devem ser utilizadas para minimizar o efeito originada da atividade laboral do CD (Carmo et al. 2011). No presente estudo, 62\% dos entrevistados afirmaram não realizar algum tipo de atividade física e somente $54 \%$ dos CD tiveram preocupação com o planejamento do ambiente de trabalho quando se tratar de ergonomia.

A presença de sintomas musculoesqueléticos em CD é resultado da soma da má postura durante o trabalho com horas seguidas em posições desconfortáveis, mantendo normalmente os membros superiores suspensos, o tronco rotacionado e com a cabeça flexionada. Tais posições exigem muito da musculatura cervical e escapular que devido à falta de apoio, sustenta o membro superior e as regiões torácicas e lombar. Entre os principais resultados verificados no presente estudo, 55\% dos participantes relataram não ter conhecimentos acerca do DORT, tendo em vista que essas alterações têm caráter cumulativo e progressivo, é importante salientar a necessidade de implementação de programas de educação em saúde, com intuito de minimizar os dados causados pelo exercício dessa profissão (Silva et al. 2013). 


\section{Conclusão}

A partir dos resultados descritivos e preliminares do presente estudo, os sintomas de DORT parecem se iniciar ainda na graduação, sendo os sintomas das regiões cervical e lombar os mais relatados por profissionais e estudantes de odontologia. Portanto, é importante que sejam implantados programas de educação que auxiliem no conhecimento de DORT já na graduação. Para melhor compreensão da relação causa e efeito entre as jornadas do CD e dos estudantes de odontologia, é fundamental que estudos com desenho longitudinal sejam realizados.

\section{Referências}

Aguiar, C., Neves, F. C. \& Araújo, M. M. (2014). O ambiente e as doenças do trabalho: percepção dos principais sintomas de desconforto/dor, relacionados aos aspectos ergonômicos na prática odontológica. Revista Tecnologia \& Informação 1, 7-20.

Barbosa, R. E. C., Assunção, A. A., \& Araújo, T. M. (2012). Distúrbios musculoesqueléticos em trabalhadores do setor saúde de Belo Horizonte, Minas Gerais, Brasil. Caderno de Saúde Pública 28, 1569-1580.

Borges, A. B., Pucci, C. R., \& Torres, C. R. G. (2009). Evaluation of the occupational health program applied the students of dentistry. Brazilian Dental Science $12,59-63$.

Carmo, I. V., Soares, E. A., Junior, V., Sidra, J., \& Guerra, R. O. (2011). Fatores associados à sintomatologia dolorosa e qualidade de vida em odontólogos da cidade de Teresina - PI. Revista Brasileira de Epidemiologia 14, 141-150.

Carvalho, R. L. R., Brasileiro, F. R. C. P., \& Lima, V. M. C. (2019). Demandas psicológicas, baixo apoio social e repetitividade: fatores ocupacionais associados à dor musculoesquelética de trabalhadores da indústria de calçados. Revista Brasileira de Saúde Ocupacional 44, e6.

Casarin, C. A. S., \& Caria, P. H. F. (2008). Comportamento muscular durante diferentes práticas odontológicas. Ciência Odontológica Brasileira 11, 64-70.

Diaz-Caballero, A. J., Goméz-Palencia, I. P., \& Díaz-Cárdenas, S. (2010). Ergonomic factors that cause the presence of pain muscle in students of dentistry. Medicina Oral, Patologia Oral y Cirurgia Bucal 6, 906-11.

Hämmig, O., \& Bauer, G. F. (2014). Work, work-life conflict and health in an industrial work environment. Occupational Medicine 64, 34-38.

Khan, S. A., \& Chew, K. Y. (2013). Effect of working characteristics and taught ergonomics on the prevalence of musculoskeletal disorders amongst dental students. BMC Musculoskeletal Disorders 14, 114-118.

Marques, A. P., Assumpção, A., de Sousa, A., Matsutani, L., Akemi, L., \& Lage, L. V. (2007). Exercícios de alongamento ativo em pacientes com fibrimialgia: efeitos no sintoma e na qualidade de vida. Fisioterapia e Pesquisa 14, 18-24.

Mascarenhas, C. H. M., \& Novaes, S. V. (2015). Sintomas osteomusculares em acadêmicos dos cursos de saúde de uma universidade pública. C\&D-Revista Eletrônica da Fainor 8, 113-131.

Mattos, J. G. S.; Castro, S. S.; Melo, L. B. L.; Santana, L. C.; Coimbra, M. A. R.; \& Ferreira, L. A. (2021): Musculoskeletal pain and perceived stress by teachers during the COVID-19 pandemic. Research, Society and Development 10, e25110615447.

Medeiros, U. V., \& Segatto, G. G. (2012). Lesões por esforços repetitivos (LER) e distúrbios osteomusculares (DORT) em dentistas. Revista Brasileira de Odontologia 69, 49-54.

Ministério da Saúde. (2012). Dor relacionada ao trabalho: lesões por esforços repetitivos (LER): distúrbios osteomusculares relacionados ao trabalho (Dort). Secretaria de Vigilância em Saúde. Departamento de Vigilância em Saúde Ambiental e Saúde do Trabalhador. Brasília, DF: Ministério da Saúde.

Pinheiro, F. A., Tróccoli, B. T., \& Carvalho, C. V. (2002). Validação do Questionário Nórdico de Sintomas Osteomusculares como medida de morbidade. Revista de Saúde Pública 3, 307-312.

Regis Filho, G. L., Michels, G., \& Sell, I. (2006). Lesões por Esforços Repetitivos e Distúrbios Osteomusculares em Cirurgiões - Dentistas. Revista Brasileira de Epidemiologia 9, 346-359.

Rosenberg, M. S. (2005). Cuidados que o dentista deve ter com sua postura. Uniodonto Leste Fluminense, [online]. http//www.google.com.br. Acesso em $[05 / 06 / 21]$.

Santos, I. N.; Martins, I. C.; Santos, A. C.; Oliveira, A. S.; Brasileiro-Santos, M. S. (2020). Distúrbios osteomusculares relacionados ao trabalho: uma caracterização da sintomatologia e dos exames diagnósticos. Research, Society and Development 10, e36710111865.

Silva, H. P. L., \& Jesus, C.S. (2013): Sintomas osteomusculares em cirurgiões-dentistas da rede pública. Revista da Amrigs 57, 44-48.

Silva, A. F. M., Campos, A. P. O., Nunes, K. A. S., Bertoncello, D., \& Walsh, I. A. P. (2013). Percepção do estado de saúde, sintomas emocionais e musculoesqueléticos em cirurgiões-dentistas. Conscientiae Saúde 12, 455-462.

Silva, J. F., Silva, H. F., Granadeiro, D. S., Raimundo, D. D., Vieira, G. C., Granadeiro, R. M. A., Hanzelmann, R. S., \& Passos, J.P. (2020). Osteomuscular symptoms related to work: implications for nursing. Research, Society and Development 9, e356997237. 
Research, Society and Development, v. 10, n. 7, e51210716891, 2021

(CC BY 4.0) | ISSN 2525-3409 | DOI: http://dx.doi.org/10.33448/rsd-v10i7.16891

Siqueira, G. R., Silva, A. M., Vieira, R. A. G., \& Silva, R. B. (2010). Dores músculo-esqueléticas em estudantes de odontologia. Revista Brasileira em Promoção da Saúde 23, 150-159.

Sousa, F. C. A.; Miranda Júnior, R. N. C.; Siqueira, H. D. S.; Silva, W. C.; Sousa, J. P.S.; \& Pinho, R.S. (2020). Behavior of biomarkers in public high school teachers. Research, Society and Development 9 , e64911616. 